\title{
A SECOND CATEGORY SET WITH ONLY FIRST CATEGORY FUNCTIONS
}

\author{
P. KOMJÁTH
}

(Communicated by Andreas R. Blass)

\begin{abstract}
If the existence of a measurable cardinal is consistent then it is consistent in that there is a second category set $A \subseteq R$ such that every $A \rightarrow A$ function, as a subset of $R^{2}$, is of first category. Some other connected results are also proved.
\end{abstract}

\section{INTRODUCTION}

P. Erdös and the author proved in [4] that the plane admits a decomposition into countably many pieces none containing the three nodes of a right-angled triangle if and only if the continuum hypothesis holds. This implies that if the $\mathrm{CH}$ holds then there exists a second category set omitting right-angled triangles. The latter statement can actually be proved without any additional hypothesis by a transfinite recursion of length $2^{\omega}$. One only has to observe that $<2^{\omega}$ lines and circles cannot cover a residual set, that is, the complement of a first category set. In fact this idea can be used to show that even in $R^{n}$, there exist second category/positive outer measure sets omitting rational distances, triangles of given shape/angle, or isosceles triangles, or any predetermined geometrical configurations. Returning to the result in [4], it implies the stronger statement, that (if $\mathrm{CH}$ holds) every second category set in $R^{2}$ contains a second category subset, omitting right-angled triangles. We show that this last statement is independent. For this, we prove that, assuming the consistency of a measurable cardinal, it is consistent that there exists a second category set $A \subseteq R$, such that the graph of every $f: A \rightarrow A$ function is of first category, as a subset of $R^{2}$ (it is convenient to identify functions with their graphs, as is done in axiomatic set theory). The measurable cardinal is not needed for the case of one-to-one functions. We show that for the general case at least an inaccessible cardinal is needed.

Using similar techniques, we prove a result complementary to an old theorem of Ulam; if the existence of a huge cardinal is consistent, then so is the existence of a second category set $A \subseteq R$ of size $\omega_{3}$ such that every smaller subset is

Received by the editors January 9, 1990 and, in revised form, August 8, 1990.

1980 Mathematics Subject Classification (1985 Revision). Primary 03E15; Secondary 03E35, $04 \mathrm{~A} 15,54 \mathrm{H} 05$. 
of first category and $A$ cannot be decomposed into $\omega_{3}$ many disjoint second category sets.

We use the standard set theory notions (see e.g. $[5,8]$ ). Cardinals are identified with initial ordinals. For a set $X, \kappa$ a cardinal,

$$
\begin{aligned}
{[X]^{\kappa} } & =\{Y \subseteq X:|Y|=\kappa\}, \\
{[X]^{<\kappa} } & =\{Y \subseteq X:|Y|<\kappa\} .
\end{aligned}
$$

\section{ONE-TO-ONE FUNCTIONS}

Theorem 1. It is consistent that $2^{\omega}=\omega_{2}$ and there exists a second category set $A \subseteq R$ such that every one-to-one function $f: A \rightarrow A$ is of first category.

Proof. Let $V$ be a model of $\mathrm{ZFC}+\mathrm{GCH}$. Let $P$ add $\omega_{1}$ Cohen reals. Let $A=\left\{r_{\alpha}: \alpha<\omega_{1}\right\}$ be the set of those Cohen reals. If $N \subseteq \omega_{1}$ is a nonstationary subset, let $Q(N)$ be the canonical partial ordering (see [5]) making $\left\{r_{\alpha}: \alpha \in N\right\}$ of first category. Let $Q$ be the finite support product of these $Q(N)$ 's. Our model will be $V^{P \star Q}$. It is well known that $P, P \star Q$ are ccc.

We first show that every one-to-one $f: A \rightarrow A$ is of first category. Let $g: \omega_{1} \rightarrow \omega_{1}$ be the function such that $f\left(r_{\alpha}\right)=r_{g(\alpha)}$. By ccc, there is a closed unbounded set $C \subseteq \omega_{1}$ such that if $\alpha<\gamma \in C$, then $g(\alpha), g^{-1}(\alpha)<\gamma$ is forced by 1 . If $N=\omega_{1}-C$, then the graph of $f,\left\{\left\{r_{\alpha}, f\left(r_{\alpha}\right)\right\}: \alpha<\omega_{1}\right\}$ is covered by

$$
\left(\left\{r_{\alpha}: \alpha \in N\right\} \times R\right) \cup\left(R \times\left\{r_{\alpha}: \alpha \in N\right\}\right) \cup\{(x, x): x \in R\},
$$

and these sets are of first category.

We finally show that $A$ is of second category in $V^{P \star Q}$. Assume that 1 forces $A$ to be covered by $F_{0}, F_{1}, \ldots$, some nowhere dense, closed sets. Every nowhere dense, closed set can be described as the complement of some union of rational intervals. There are, by ccc, in $V^{P}$, countably many conditions $q_{i} \in Q \quad(i=0,1, \ldots)$ completely determining the $F_{i}$ 's. There are in $V$ nonstationary sets $N_{0}, N_{1}, \ldots$ such that the supports of the $q_{i}$ 's are covered by $\left\{N_{0}, N_{1}, \ldots\right\}$. Select a $\gamma \in \omega_{1}-\left(N_{0} \cup N_{1} \cup \cdots\right)$. We show that $1 \Vdash r_{\gamma} \notin \bigcup F_{i}$, and this gives the desired contradiction. It suffices to show that if $(p, q) \in P \star Q$, $i<\omega$, then there is an extension of $(p, q)$ forcing that $r_{\gamma} \notin F_{i}$. Assume that the information $p$ gives on $r_{\gamma}$ is that $r_{\gamma} \in E$, where $E$ is a diadic interval. In $V^{P}$ it is true that there is an extension of $q$ in $Q$ forcing that $F_{i} \cap E^{\prime}=\varnothing$ for some diadic subinterval $E^{\prime}$ of $E$. By the above remarks, we can find such a condition among those with supports from $\left\{N_{0}, N_{1}, \ldots\right\}$. Back in $V$ we can find $\left(p^{\prime}, q^{\prime}\right)$ forcing $F_{i} \cap E^{\prime}=\varnothing$ where the $\gamma$ th coordinate of $p^{\prime}$ is still $E$, otherwise $p^{\prime}$ extends $p$. If we extend $\left(p^{\prime}, q^{\prime}\right)$ to a condition $\left(p^{\prime \prime}, q^{\prime}\right)$ where the only change is that the $\gamma$ th coordinate of $p^{\prime \prime}$ is $E^{\prime}$, then $\left(p^{\prime \prime}, q^{\prime}\right)$ forces that $r_{\gamma}$ is in the empty interval $E^{\prime} \cap F_{i}$ which is impossible. 
An easy argument gives

Theorem 2. If $\mathrm{CH}$ holds, then every second category set has a second category one-to-one function onto itself.

Proof. Assume that $A \subseteq R$ is a second category set.

Claim. $A \times A$ is of second category.

Proof of Claim. Assume that $A \times A=\bigcup\left\{T_{n}: n<\omega\right\}$, with $T_{n}$ nowhere dense. Put $S(x, n)=\left\{y \in A:(x, y) \in T_{n}\right\}$ for $x \in A$. As $\bigcup\{S(x, n): n<\omega\}=A$, for every $x \in A$, some $S(x, n)$ is dense in some rational interval $I$. If $n<\omega$, $I$ is a rational interval, put $U(n, I)=\{x \in A: S(x, n)$ is dense in $I\}$. As $\cup\{U(n, I): n, I\}=A$, for some $n, I, U(n, I)$ is dense in an interval $J$, so $T_{n}$ is dense in $I \times J$, a contradiction.

Enumerate the first category $F_{\sigma}$ subsets of $R^{2}$ as $\left\{B_{\alpha}: \alpha<\omega_{1}\right\}$. Select by transfinite recursion $a_{\alpha}, b_{\alpha} \in A$ such that $a_{\alpha} \neq a_{\beta}, b_{\alpha} \neq b_{\beta} \quad(\beta<\alpha)$ and $\left(a_{\alpha}, b_{\alpha}\right) \notin B_{\alpha}$. As $A \times A$ is of second category, countably many lines plus $B_{\alpha}$ cannot cover it, so $a_{\alpha}, b_{\alpha}$ may be selected. If we set $f\left(a_{\alpha}\right)=b_{\alpha}$, then $f$ is not covered by any $B_{\alpha}$, so it is of second category.

\section{GeNERAL FUNCTIONS}

Theorem 3. If the existence of a measurable cardinal is consistent then so is the existence of a second category set $A \subseteq R$ such that every $A \rightarrow A$ function is of first category. The same holds for every $A^{n} \rightarrow A$ function $(n=1,2, \ldots)$.

Proof. Let $V$ be a model of $Z F C$ such that $\kappa$ is measurable, and $I$ is a $\kappa$-complete, normal, prime ideal on $\kappa$. As in the proof of Theorem 1 , let $P$ add $\kappa$ Cohen reals, let their set be $A=\left\{r_{\alpha}: \alpha<\kappa\right\}$, and let $Q$ make every $\left\{r_{\alpha}: \alpha \in B\right\} \quad(B \in I)$ of first category. As is the proof of Theorem $1, P * Q$ is ccc and $A$ is of second category, even in $V^{P, Q}$.

Assume first that $f: A \rightarrow A$ is a function in $V^{P, Q}$. Let $\underline{f}$ be a name for $f$ such that $1 \Vdash \underline{f}: A \rightarrow A$. By ccc for every $\alpha<\kappa$ the set of potential values

$$
T_{\alpha}=\left\{\xi<\kappa: \exists p \Vdash f\left(r_{\alpha}\right)=r_{\xi}\right\}
$$

is countable. Say $T_{\alpha}=\left\{g_{n}(\alpha): n<\omega\right\}$. It suffices to show that $S\left(g_{n}\right)$ is of first category, where $S(g)=\left\{\left(r_{\alpha}, r_{g(\alpha)}\right): \alpha<\kappa\right\}$. If $g: \kappa \rightarrow \kappa, g \in V$ is given, by the properties of $I$, either $g(\alpha)=c$ holds for some $c<\kappa$, outside a set in $I$, which will be abbreviated by $g(\alpha)=c$ almost everywhere, or $g(\alpha)=\alpha$ almost everywhere, or else $g(\alpha)>\alpha$ almost everywhere. Let $B$ be the set of those $\alpha$ values for which the appropriate (in) equality does not hold. As $B \in I$, $C=\left\{r_{\alpha}: \alpha \in B\right\}$ is of first category, in the final model.

The range of $g$ is in $I$, in the first and the third cases. This is obvious for the first case, but in the third case there is a regressive function, namely $g^{-1}$ on it, which does not have an unbounded set where it is constant. The normality of $I$ gives the statement. 
This implies that $D=\left\{r_{g(\alpha)}: \alpha<\kappa\right\}$ is of first category in $V^{P, Q}$. The graph in question is covered by

$$
(C \times R) \cup(R \times\{c\}), \quad(C \times R) \cup\{(x, x): x \in R\}, \quad \text { and } \quad(C \times R) \cup(R \times D),
$$

in the respective cases, so it is of first category.

To prove the general result about $f: A^{n} \rightarrow A$, it again suffices to show that if $g: \kappa^{n} \rightarrow \kappa$, then $T(g)=\left\{\left(r_{x_{1}}, \ldots, r_{x_{n}}, r_{g\left(x_{1}, \ldots, x_{n}\right)}\right): x_{i}<\kappa\right\}$ is of first category in $R^{n+1}$. It is well known that there exists a set $B \in I$ such that $\kappa-B$ is a set of indiscernibles (see [5]). If $\pi:\{1,2, \ldots, n\} \rightarrow\{1,2, \ldots, n\}$ is a permutation, then $y=g\left(x_{\pi(1)}, \ldots, x_{\pi(n)}\right)$ lies in the same interval (depending only on $\pi$ ) with respect to $x_{1}<\cdots<x_{n}$ for $x_{1}, \ldots, x_{n} \in \kappa-B$. This implies that $y \in B$. Therefore, every vector in $T(g)$ has a coordinate in $\left\{r_{\alpha}: \alpha \in B\right\}$, which is of first cateogry, and so is $T(g)$.

Corollary 4. If the existence of a measurable cardinal is consistent then so is the existence of a second categcry planar set that does not contain a second category subset omitting right angled triangles.

Proof. $A \times A$ from Theorem 3 will work. Assume that $B \subseteq A \times A$ spans no right-angled triangles. Then, for $(x, y) \in B$ there is either no $z \in A$ such that $z \neq y$ and $(x, z) \in B$, or else there is no $z \in A, z \neq x$ such that $(z, y) \in B$. In other words, $B$ is covered by a 'horizontal' and by a 'vertical' function.

Statement 5. Corollary 4 is true with 'rectangles' in place of 'right-angled triangles.'

Proof. We use the model of Theorem 3. Assume that $1 \Vdash B \subseteq A \times A$. For $\alpha<\beta<\kappa$, either $1 \Vdash\left(r_{\alpha}, r_{\beta}\right),\left(r_{\beta}, r_{\alpha}\right) \notin B$ or there is a $p(\alpha, \beta)$ forcing $\left(r_{\alpha}, r_{\beta}\right) \in B$ or forcing $\left(r_{\beta}, r_{\alpha}\right) \in B$. Fix such a $p(\alpha, \beta)$ (if it exists). If $\alpha<$ $\beta_{1}<\beta_{2}$ and $p\left(\alpha, \beta_{1}\right), p\left(\alpha, \beta_{2}\right)$ are compatible, fix a common lower bound $p\left(\alpha, \beta_{1}, \beta_{2}\right)$. If, for $\alpha_{1}<\alpha_{2}<\beta_{1}<\beta_{2}, p\left(\alpha_{1}, \beta_{1}, \beta_{2}\right)$ and $p\left(\alpha_{2}, \beta_{1}, \beta_{2}\right)$ are compatible, fix $q\left(\alpha_{1}, \alpha_{2}, \beta_{1}, \beta_{2}\right)$, a common lower bound.

Color the pairs, triples, and quadruples of $\kappa$ according to which of the abovementioned cases occur. By measurability, there is a measure one set $Z \subseteq \kappa$ on which the same cases hold. If, for $\alpha<\beta$ in $Z, 1$ forces $\left(r_{\alpha}, r_{\beta}\right),\left(r_{\beta}, r_{\alpha}\right) \notin$ $B$, then $B$ is covered by two first category sets as in the previous proofs, and we are done. We can therefore assume that, say, $p(\alpha, \beta)$ forces $\left(r_{\alpha}, r_{\beta}\right) \in B$ for $\alpha<\beta$ in $Z$. By the homogeneity of $Z$, either $p\left(\alpha, \beta_{1}\right), p\left(\alpha, \beta_{2}\right)$ are incompatible for $\alpha<\beta_{1}<\beta_{2}$ in $Z$, or they are always compatible. The former case is impossible, as the applied notion of forcing is ccc, and there are $\omega_{1}$ different elements above an $\alpha$ in $Z$. Again, by homogeneity, either $p\left(\alpha_{1}, \beta_{1}, \beta_{2}\right), p\left(\alpha_{2}, \beta_{1}, \beta_{2}\right)$ are incompatible whenever $\alpha_{1}<\alpha_{2}<\beta_{1}<\beta_{2}$ in $Z$, or else they are always compatible. The former case is impossible, as there are $\beta_{1}<\beta_{2}$ in $Z$, preceded by $\omega_{1}$ elements of $Z$, and the notion of forcing is ccc. There are, therefore, $\alpha_{1}<\alpha_{2}<\beta_{1}<\beta_{2}$ such that $q\left(\alpha_{1}, \alpha_{2}, \beta_{1}, \beta_{2}\right)$ exists, and forces the rectangle $\left\{r_{\alpha_{1}}, r_{\alpha_{2}}\right\} \times\left\{r_{\beta_{1}}, r_{\beta_{2}}\right\}$ to be in $B$. 
We notice that if a set $A$ satisfies Theorem 3, then $|A|>\omega_{1}$. For this, one takes the Sierpinski decomposition of $A \times A$ into countably many 'horizontal' and 'vertical' functions. One of them is of second category. (See e.g. $[10,11]$.)

It is possible that the conclusion of Theorem 3 implies the consistency of a measurable cardinal. Although we have not been able to prove this we have some evidence in its favor.

If $\kappa$ is a cardinal, an ultrafilter $U$ over $\kappa$ is uniform, if $|A|=\kappa$ holds for every $A \in U$. An ultrafilter $U$ is $(\omega, \kappa)$-regular, if there exist $A_{\alpha} \in U$ $(\alpha<\kappa)$, such that the intersection of any infinitely many $A_{\alpha}$ 's is empty.

Statement 6. If a set as in Theorem 3 exists, then there is for some $\kappa$ a uniform, non- $(\omega, \kappa)$-regular ultrafilter over $\kappa$.

Proof. Assuming that $A \subseteq R$ satisfies Theorem 3, we can assume that $\kappa=|A|$ is of minimal cardinality. Then every subset of $A$ of size $<\kappa$ is of first category. Put $I=\{B \subseteq A: B$ is of first category $\}$. $I$ is clearly a nontrivial, $\omega_{1}$-complete ideal.

Claim 1. There are no mutually disjoint sets $B_{\alpha} \notin I, B_{\alpha} \subseteq A, \alpha<\kappa$.

Proof. Assume that $\left\{B_{\alpha}: \alpha<\kappa\right\}$ are disjoint, $A=\left\{a_{\alpha}: \alpha<\kappa\right\}$. Define $f: A \rightarrow A$ as follows: $f(x)=a_{\alpha}$ if $x \in B_{\alpha}$. If the graph of $f$ is of first category, then by the Fubini theorem, for all but first category many $y \in R$, the set $\{x \in R: f(x)=y\}$ is of first category. There is, therefore, a $a_{\alpha} \in A$ such that $B_{\alpha}=f^{-1}\left(a_{\alpha}\right)$ is of first category; a contradiction.

To conclude, we need an argument of Silver (see [1]). Let $I$ be an $\omega_{1^{-}}$complete ideal over some $\kappa, I \supseteq[\kappa]^{<\kappa} . I^{+}=\{X \subseteq \kappa: X \notin I\}, I^{\star}=\{X \subseteq$ $\kappa: \kappa-X \in I\}$.

Claim 2 (Silver). If there are no $\kappa$ disjoint sets in $I^{+}$, then every ultrafilter $U \supseteq I^{\star}$ is $(\omega, \kappa)$-irregular.

Proof. Assume that $X_{\alpha} \in U(\alpha<\kappa)$. Put $Y \in G$ if there exists some $s \in[\kappa]^{<\kappa}$ such that $X_{\alpha}-Y \in I$ for all $\alpha \notin s$. Obviously, $\varnothing \notin G$.

Subclaim. $G$ is $\omega_{1}$-complete.

Proof. Assume that $Y_{n} \in G, s_{n}, n=0,1, \ldots$, witness this. Then $s=$ $\bigcup\left\{s_{n}: n<\omega\right\}$ witnesses that $Y=\bigcap\left\{Y_{n}: n<\omega\right\} \in G$. If $\alpha \notin s$, then $\alpha \notin s_{n}$ $(n<\omega)$, so $X_{\alpha}-Y \subseteq \bigcup\left\{\left(X_{\alpha}-Y_{n}\right): n<\omega\right\} \in I$, as $I$ is $\omega_{1}$-complete. As $[\kappa]^{<\kappa} \subseteq I$, and $I$ is $\omega_{1}$-complete, of $(\kappa)>\omega$, so $|s|<\kappa$.

Select $\alpha(\xi)<\kappa$ as long as possible (say for $\xi<\theta$ ), such that $X_{\alpha(\xi)}-$ $\bigcup\left\{X_{\alpha(\tau)}: \tau<\xi\right\} \in I^{+}$. By our hypotheses, $\theta<\kappa$. Let $S_{0}=\{\alpha(\xi): \xi<\theta\}$, then from $\kappa-S_{0}$ select similarly $S_{1}$, then $S_{2}$, etc. By the definition of $G$, $Y_{n}=\bigcup\left\{X_{\alpha}: \alpha \in S_{n}\right\} \in G$. Select $\gamma \in \bigcap\left\{Y_{n}: n<\omega\right\}$, then $\alpha_{n} \in S_{n}$ such that $\gamma \in X_{\alpha_{n}}$. We are done, as $\bigcap\left\{X_{\alpha_{n}}: n<\omega\right\} \neq \varnothing$. 
H.-D. Donder obtained several results on nonregular ultrafilters [2]. Though he only proved that the existence of an ultrafilter as in Claim 2 implies the consistency of an inaccessible cardinal, it is very likely that those methods will lead to the proof of the consistency of a measurable cardinal, and giving the necessary counterpart to Theorem 3 .

\section{DeCOMPOSITIONS OF SECOND CATEGORY SETS}

An old result of Ulam [12] says that if

$$
\text { no cardinal } \leq 2^{\omega} \text { is weakly inaccessible }
$$

then every second category set is the disjoint union of $\omega_{1}$ second category sets. We show in [6] that this statement is not true in general. Ulam's argument actually shows that under (*) every second category $A \subseteq R$ is the disjoint union of $\tau$ second category sets, where $\tau$ is the least cardinal such that the union of $\tau$ many first category subsets of $A$ may be of second category. Obviously, $\tau$ is uncountable and regular. One can ask if under $(*), \tau$ can be replaced by $\theta=\min \{|B|: B \subseteq A$ 2nd category $\}$. We show that it is not the case.

Theorem 7. If the existence of a huge cardinal is consistent then so is that $2^{\omega}=$ $\omega_{4}$ and there exists a second category set $A \subseteq R$ with $|A|=\omega_{3}$, all $B \subseteq A$ with $|B| \leq \omega_{2}$ are of first category, and $A$ is not the union of $\omega_{3}$ disjoint second category sets.

Proof. We are going to build a model similar to those in Theorems 1 and 3. Let $I$ be a uniform ideal on $\omega_{3}$ such that

$$
I \text { is } \omega_{1} \text { - complete; }
$$

$$
\begin{aligned}
& \text { if } A_{\xi} \in I^{+}\left(\xi<\omega_{3}\right) \text {, then there is a } Z \in\left[\omega_{3}\right]^{\omega_{1}} \text { such that } \\
& \bigcap\left\{A_{\xi}: \xi \in Z\right\} \neq \varnothing .
\end{aligned}
$$

Then let $P$ be the notion of forcing which adds $\omega_{3}$ Cohen reals, $\left\{r_{\alpha}: \alpha<\right.$ $\left.\omega_{3}\right\}$. In $V^{P}$, for $B \in I$, let $Q(B)$ be the canonical notion of forcing that makes $\left\{r_{\alpha}: \alpha \in B\right\}$ of first category. $Q$ is the finite support product of the $Q(B)$ 's for all $B \in I$. We force by $P^{*} Q$. The proof that $A=\left\{r_{\alpha}: \alpha<\omega_{3}\right\}$ remains of second category is entirely the same as the corresponding proof in Theorem 1.

Assume that 1 forces $\left\{A_{\xi}: \xi<\omega_{3}\right\}$ to be a decomposition of $A$. Take $B_{\xi}=\left\{\alpha<\omega_{3}: \exists p \Vdash r_{\alpha} \in A_{\xi}\right\}$. If some $B_{\xi}$ is in $I$, then $A_{\xi}$ is made first category by $P \star Q$. If $B_{\xi} \notin I$ for all $\xi<\omega_{3}$, then by (3.2), there is a $Z \in\left[\omega_{3}\right]^{\omega_{1}}$ and a $\tau<\omega_{3}$ such that $\tau \in B_{\xi}$ for $\xi \in Z$. Choose $p(\xi) \in P \star Q$ forcing $r_{\tau} \in A_{\xi}$, then $\{p(\xi): \xi \in Z\}$ are $\omega_{1}$ incompatible elements of $P \star Q$, a contradiction, since $P \star Q$ is ccc.

The remainder of the proof is dedicated to showing that a model of Magidor [9] contains an ideal $I$ as described in (3.1, 3.2). Magidor's model can briefly be described as follows. Let $\kappa$ be a huge cardinal, $j: V \rightarrow M$ an elementary 
embedding with $\kappa$ as critical point, $j(\kappa)=\lambda,[M]^{\lambda} \subseteq M$. Let $P$ be a notion of forcing such that

$$
\begin{gathered}
|P|=\kappa, P \text { is } \kappa \mathrm{cc} ; \\
V^{P} \Vdash \kappa=\omega_{1} ;
\end{gathered}
$$

if $\alpha<\kappa$ is regular, $C \subseteq P$ is a regular subalgebra, $|C|<\kappa$, then $C * \operatorname{Col}(\alpha, \kappa) \subseteq P$.

A forcing notion with these properties can be built by diagonalization, see [8, 9]. In $V^{P}$, let $Q$ be the canonical poset collapsing $\lambda$ to $\kappa^{++}=\omega_{3}$ via conditions of size $\leq \kappa$. In $V^{P, Q}$ we are going to construct an ideal $I$ over $[\lambda]^{<\kappa}$. As $\mathrm{GCH}$ holds, this suffices. In $M^{j(P)}, j(Q)$ is a partial ordering, which, over $M^{j(P)}$, collapses $j(\lambda)$ to $\lambda^{++}$with conditions of size $\leq \lambda$.

In $V^{j(P)}$ we are going to define $\left\{y_{\alpha}: \alpha<\lambda^{+}\right\}$, a decreasing sequence of elements of $j(Q)$. Let $y_{0}$ be a master condition for a generic $G \subseteq j(P)$. Then for every $A \subseteq[\lambda]^{<\kappa}, A \in V^{P, Q}$ let a $y_{\alpha}$ be selected that

$$
y_{\alpha} \Vdash j^{\prime \prime} \lambda \in j(A) \text { or } y_{\alpha} \Vdash j^{\prime \prime} \lambda \notin j(A) \text {. }
$$

In $V^{j(P)}$, let $A \in \mathscr{F}$ if for some $\alpha, y_{\alpha} \Vdash j^{\prime \prime} \lambda \in j(A)$. This is an ultrafilter, in $V^{j(P)}$, over $P\left([\lambda]^{<\kappa}\right) \cap V^{P, Q}$. $\mathscr{F}$ is $\kappa$-complete, see [9].

In $V^{P, Q}$ we define $I$ as follows. $A \subseteq[\lambda]^{<\kappa}, A \in V^{P, Q}$ is in $I$ if $1 \Vdash A \in$ $\mathscr{F}$. As $\mathscr{F}$ is $\kappa$-complete, $I$ is $\kappa$-complete, so (3.1) is fulfilled.

For (3.2), let $A_{\alpha} \in I,(\alpha<\lambda)$ be given. Let $p(\alpha) \in j(P) / P * Q$ force $A \in \mathscr{F} . j(P) / P * Q$ is $\lambda \mathrm{cc}$, and a well-known lemma with the regularity of $\lambda$ ensures that some $p$ forces that $\lambda$ many $p(\alpha)$ 's are in the generic set. Forcing by this element, we get that, in $V^{j(P)}, \lambda$ many of the $A_{\alpha}$ 's are in $\mathscr{F}$. For each of them, there is a $y_{\xi}$ forcing $j^{\prime \prime} \lambda \in j\left(A_{\alpha}\right)$.

As $M$ is closed under $\lambda$-sequences, in $M^{j(P), j(Q)}$ for $j\left(A_{\alpha}: \alpha<\lambda\right)$, it is true that for $\lambda$ many elements of the sequence it is forced that $j^{\prime \prime} \lambda$ is contained in them, as $j(A)_{j(\alpha)}=j\left(A_{\alpha}\right)$ (for $\left.\alpha<\lambda\right)$. For $\lambda$ many of them, the intersection is nonempty, therefore, by the elementarity of $j$, for $\kappa$ many $A_{\alpha}$, the intersection of them is nonempty in $V^{P, Q}$.

\section{ACKNOWLEDGMENT}

The author is grateful to the referee for several remarks and suggestions.

\section{REFERENCES}

1. W. Boos, Lectures on large cardinal axioms, Lecture Notes in Math., vol. 669, SpringerVerlag, Berlin and New York, pp. 25-88.

2. H.-D. Donder, Regularity of ultrafilters and the core model, Israel J. Math. 63 (1988), 289322. 
3. P. Erdös, Set theoretic, measure theoretic, combinatorial, and number theoretic problems concerning point sets in Euclidean space, Real Analysis Exchange 4 (1978-79), 113-138.

4. P. Erdös and P. Komjáth, Countable decompositions of $R^{2}$ and $R^{3}$, Discrete Comput. Geom. 5 (1990), 325-331.

5. T. Jech, Set theory, Academic Press, New York, 1978.

6. P. Komjáth, On second-category sets, Proc. Amer. Math. Soc. 107 (1989), 653-654.

7. K. Kunen, Set theory. An introduction to independence proofs, North-Holland, Amsterdam, 1980.

8. __ Saturated ideals, J. Symbolic Logic 43 (1978), 65-76.

9. M. Magidor, On the existence of nonregular ultrafilters and the cardinality of ultrapowers, Trans. Amer. Math. Soc. 249 (1979), 97-111.

10. W. Sierpiński, Sur un théorème équivalent à l'hypothèse du continu, Bull. Acad. Polon. Lett. Sér. A (1919), 1-3.

11. J. C. Simms, Sierpinski's theorem (to appear).

12. St. Ulam, Über gewisse Zerlegungen von Mengen, Fund. Math. 20 (1933), 221-223.

Department of Computer Science, R. EÖtVös University, Budapest, Muzeum KRT 6-8, 1088 HUNGARY 